\title{
Efeito da temperatura em processos de sinterização de pós de hidroxiapatita
}

\section{(Effect of temperature in process of sintering of hydroxyapatite powders)}

\author{
A. G. de S. Azevedo, K. Strecker, H. F. Gorgulho \\ Departamento de Ciências Naturais - DCNat, Universidade Federal de S. João del-Rei - UFSJ \\ Campus Dom Bosco, Praça Dom Helvécio 74 - Fábricas, S. João del-Rei, MG 36301-160 \\ adrianogalvao26@yahoo.com.br,strecker@ufsj.edu.br,Gorgulho@ufsj.edu.br
}

\begin{abstract}
Resumo
Amostras de hidroxiapatita foram sintetizadas por meio da precipitação de soluções aquosas contendo os íons precursores. O precipitado obtido foi lavado, seco, desaglomerado e peneirado com o intuito de se obter amostras em forma de pó e com granulometria homogênea. O material foi sinterizado em diferentes temperaturas e posteriormente caracterizado por difração de raios $\mathrm{X}$, espectroscopia no infravermelho, microscopia eletrônica de varredura, análise termogravimétrica e termodiferencial e a quantificação da área superficial específica e porosidade foi realizada por meio dos métodos Brunauer-Emmett-Teller e BarretJoyner-Halenda, respectivamente. Os pós apresentaram modificações na cristalinidade, morfologia e nas propriedades texturais com o aumento da temperatura. Novas fases foram identificadas nos difratogramas de raios X devido à decomposição do material, corroborando com resultados reportados. Houve o crescimento dos cristais, aumento da cristalinidade e diminuição da área de superfície específica, comprovando que as modificações ocorreram devido aos processos de sinterização, uma vez que a força motriz para esse processo é a diminuição da área de superfície específica.

Palavras-chave: hidroxiapatita, sinterização, temperatura, decomposição.
\end{abstract}

Abstract

Samples of hydroxyapatite were synthesized by precipitation from aqueous solutions containing the precursor ions. The obtained precipitate was washed, dried, deagglomerated and sieved to obtain samples in powder form of homogeneous particle size. The material was sintered at different temperatures and subsequently characterized by X-ray diffraction, infrared spectroscopy, scanning electron microscopy, thermo-gravimetric and thermo-differential analyses and quantification of the specific surface area. Furthermore, porosity was determined using the Brunauer-Emmett-Teller and Barret-Joyner-Halenda methods, respectively. The powders showed changes in crystallinity, morphology and texture with increasing temperature. New phases were identified in the $X$-ray patterns due to decomposition of the material, corroborating other findings in the literature. Crystal growth and increased crystallinity were observed during heat treatments, while specific surface decreased, proving that the changes were due to the sintering processes.

Keywords: hydroxyapatite, sintering, temperature, decomposition.

\section{INTRODUÇÃO}

As pesquisas de materiais aplicáveis nas áreas da medicina trazem um aumento na qualidade de vida de pessoas que necessitam de tratamentos mais eficazes ou soluções para problemas graves. Pesquisas relacionadas à descoberta e melhoramento de materiais usados nas diversas áreas médicas - materiais cirúrgicos, materiais para substituições de tecidos ósseos - aumentam cada dia mais devido às necessidades e o aumento do número de enfermidades. Lesões onde partes do tecido ósseo são removidas são normalmente encontradas em acidentes. Os membros são geralmente retirados para se evitar complicações posteriores ou quando a recuperação dessas partes afetadas é impossível. Esse tipo de procedimento é comum nos hospitais do Brasil representando um grande trauma para o paciente que necessitará se adaptar a novas formas de vida, dependendo de membros artificiais que substituirão as partes lesionadas $[1,2]$. A busca por novos materiais que substituam essas partes lesionadas é motivo de novas pesquisas nas áreas de materiais [3]. Os materiais obtidos a partir dessas pesquisas podem ser metálicos, poliméricos, compósitos ou cerâmicos, entretanto, todos eles devem apresentar características essenciais como: Elevada resistência mecânica, elevada resistência à fadiga, nenhuma ou baixa toxicidade, baixa solubilidade nos fluidos corpóreos e, se possível, ter boa compatibilidade com o sistema biológico onde será implantado com o intuito de se diminuir a rejeição pós-cirúrgica [2, 4]. Vários materiais possuem características ideais para serem utilizados na construção de próteses substituintes de tecidos ósseos, porém, materiais a base de fosfato de cálcio promovem a sua regeneração (osteocondução) e são usados na confecção de próteses e enxertos [2]. 
A hidroxiapatita (HAp) é um mineral da família das apatitas e possui fórmula química $\mathrm{Ca}_{10}\left(\mathrm{PO}_{4}\right)_{6}(\mathrm{OH})_{2}$, representando uma hidroxiapatita estequiométrica quando o valor da razão $\mathrm{Ca} / \mathrm{P}$ do produto obtido for próximo de 1,667 $[5,6]$. É um mineral raramente encontrado livre na natureza, porém, é um constituinte da fase óssea em concentrações consideráveis - esse mineral compõe aproximadamente $70 \%$ da fase inorgânica dos ossos e dentes - apresentando boa biocompatibilidade em enxertos produzidos a base desse fosfato de cálcio [7]. A hidroxiapatita apresenta substituições na sua rede cristalina por íons presentes no meio em que está inserida [1]. Essa facilidade de realizar a troca dos íons da sua estrutura por íons em soluções aquosas favorece sua utilização como matriz adsorvente de íons contaminantes de efluentes líquidos [8]. Concentrações elevadas desses contaminantes alteram algumas propriedades desse material [8]. As substituições na estrutura da hidroxiapatita promovem modificações no seu arranjo cristalino, as quais modificam os parâmetros da célula unitária, solubilidade e, consequentemente, a taxa de aceitação do material dentro do corpo humano. Alguns estudos têm demonstrado que a presença de íons carbonato com concentrações próximas de 7-8\% em peso promovem uma maior bioatividade das matrizes cerâmicas utilizadas em processos de osteocondução $[9,10]$. Essas substituições podem ocorrer por meio da substituição dos íons carbonato, os quais podem ocupar as posições dos grupos fosfatos $\left(\mathrm{PO}_{4}^{3-}\right)$ e hidróxidos $\left(\mathrm{OH}^{-}\right)$dentro da hidroxiapatita, formando hidroxiapatita carbonatada do tipo B e do tipo A respectivamente $[1,11,10]$. A presença dos íons carbonato pode ocorrer também por meio da substituição dos grupos $\left(\mathrm{PO}_{4}^{3-}\right)$ e $\left(\mathrm{OH}^{-}\right)$simultaneamente, formando, dessa forma, a hidroxiapatita carbonatada do tipo $\mathrm{AB}[10,11]$. A inserção de $\mathrm{CO}_{3}{ }_{3}^{2-}$ pode promover o início do processo de sinterização em temperaturas mais baixas, ocasionando a consolidação e obtenção da matriz cerâmica em menores temperaturas $[12,13]$. Porém, a presença de íons carbonato na estrutura da hidroxiapatita apenas influência na sinterabilidade do pó quando ocorre a formação de HAp do tipo B. A substituição dos íons $\mathrm{OH}^{-}$pelos $\mathrm{CO}_{3}^{2-}$ não altera o processo de sinterização da hidroxiapatita [12].

Em processos de remoção de fluoretos de efluentes pela HAp, ocorre a substituição dos íons hidróxidos pelos fluoretos $\left(\mathrm{F}^{-}\right)$ocasionando a formação da fluorapatita $\left(\mathrm{Ca}_{10}\left(\mathrm{PO}_{4}\right)_{6} \mathrm{~F}_{2}\right)$ (HAp-F), a qual possui uma constante de estabilidade maior do que a da hidroxiapatita, (HAp $\log \mathrm{K}=40,9)(\mathrm{HAp}-\mathrm{F} \log \mathrm{K}=54,6)$ [14]. A inserção de íons $\mathrm{Pb}^{2+}$ na estrutura da HAp promove o aparecimento da piromorfita $\left(\mathrm{Pb}_{10}\left(\mathrm{PO}_{4}\right)_{6}(\mathrm{OH})_{2}\right)$. Esse processo de substituição iônica entre $\mathrm{Ca}^{2+} \mathrm{ePb}^{2+}$ promove a diminuição da solubilidade do material. Essa propriedade torna a hidroxiapatita uma matriz adsorvente com grande potencial de adsorção de metais pesados por meio da troca iônica [15].

A utilização da HAp contaminada como substituinte ósseo pode comprometer todo o processo de osteocondução, necessitando posteriormente a realização de cirurgias de reparo das próteses instaladas, o que representa um aumento nos custos hospitalares e um transtorno para o paciente [2]. Várias rotas de síntese da hidroxiapatita estão disponíveis, podendo se alterar a rota para a obtenção de cristais com tamanhos diferentes ou até razões $\mathrm{Ca} / \mathrm{P}$ diferentes da estequiométrica [2, 16-20].

Os métodos de síntese mais utilizados são os de precipitação por via úmida, sol gel, hidrotermal, fusão de sais precursores em temperaturas elevadas, entre outros [21]. Dos métodos citados, a co-precipitação das soluções contendo os íons precursores é a rota de mais fácil aplicação e menor custo operacional, sendo muitas vezes o método mais viável para a produção daHAp [22, 23]. Geralmente, após a obtenção da hidroxiapatita, um posterior tratamento térmico é necessário para que a consolidação das reações, rearranjos estruturais, densificação do material, aumento da resistência mecânica e aumento da cristalinidade possam ocorrer com a consequente diminuição da área superficial específica (ASE) [12, 24-26]. O estudo das alterações estruturais devido as diferentes rotas já foi descrito por alguns autores; entretanto, as modificações sofridas pelos pós devido as diferentes temperaturas de sinterização ainda são parâmetros que devem ser avaliados para esse tipo de material em escala nanométrica $[12,27-$ 30].

Este trabalho teve como objetivo sintetizar pós de hidroxiapatita em escala nanométrica e avaliar as modificações cristalográficas, morfológicas, texturais e elementares ocorridas com o aumento da temperatura de sinterização. Temperaturas de 900, 1000, 1200 e 1300 ${ }^{\circ} \mathrm{C}$ foram escolhidas para os tratamentos térmicos. Alterações na cristalinidade, no tamanho de cristalitos, na área de superfície específica, no diâmetro médio e no volume dos poros, nas espécies presentes na matriz cerâmica, na morfologia dos grãos obtidos e na estabilidade térmica foram avaliadas nas diferentes temperaturas de sinterização. $O$ estudo do processo de sinterização da hidroxiapatita possibilitará a obtenção de informações acerca das modificações ocorridas após o tratamento térmico. $\mathrm{O}$ conhecimento das alterações físicas e químicas que ocorrem na matriz cerâmica possibilita sua melhor utilização, seja nas áreas médicas como substituinte ósseo ou como matriz adsorvente em processos de remediação ambiental.

\section{MATERIAIS E MÉTODOS}

A hidroxiapatita foi obtida por meio da co-precipitação de soluções aquosas contendo os íons precursores. Soluções contendo 1,0 e 0,6 mol. $\mathrm{L}^{-1}$ de $\mathrm{CaCl}_{2} \cdot 2 \mathrm{H}_{2} \mathrm{O}$ e $\left(\mathrm{NH}_{4}\right)_{2} \mathrm{HPO}_{4}$ (Merck, P.A.), respectivamente, foram preparadas com água Miliq- ${ }^{\circledR}$ para se evitar a contaminação do material com outros íons indesejados durante a síntese. A síntese foi realizada dentro de uma capela, livre de contaminantes externos e com o gotejamento da solução contendo o íon fosfato controlado em $\sim 5 \mathrm{~mL} \cdot \mathrm{min}^{-1}$ [19]. O becker onde a reação ocorreu foi vedado com um filme plástico (pvc) para evitar ao máximo a entrada de $\mathrm{CO}_{2}$ atmosférico e, 
consequentemente, favorecer a formação do $\mathrm{CO}_{3}{ }^{2-}$ durante a co-precipitação. A reação ocorreu em meio alcalino, em que a formação de cristais de hidroxiapatita é favorecida $[19,20,31]$. O pH das soluções foi controlado entre 9,0 e 10,0 por meio da adição de solução de hidróxido de amônio $\left(\mathrm{NH}_{4} \mathrm{OH}\right)-30-32 \%$ e a solução foi mantida em agitação (100 $\mathrm{rpm})$ e temperatura constante $\left(60^{\circ} \mathrm{C}\right)$ durante todo o período de gotejamento do $\left(\mathrm{NH}_{4}\right)_{2} \mathrm{HPO}_{4} \mathrm{em} \mathrm{CaCl}$. Após a adição das soluções, o precipitado formado foi deixado em agitação por mais $2 \mathrm{~h}$. A equação que governa a reação de obtenção da hidroxiapatita é a seguinte [22, 32]:

$$
\begin{aligned}
& 6\left(\mathrm{NH}_{4}\right)_{2} \mathrm{HPO}_{4(\mathrm{aq})}+10 \mathrm{CaCl}_{2(\mathrm{aq})}+8 \mathrm{NH}_{4} \mathrm{OH}_{(\mathrm{aq})} \rightarrow \\
& \mathrm{Ca}_{10}\left(\mathrm{PO}_{4}\right)_{6}(\mathrm{OH})_{2(\mathrm{~s})}+20 \mathrm{NH}_{4} \mathrm{Cl}_{(\mathrm{aq})}+6 \mathrm{H}_{2} \mathrm{O}_{(\mathrm{l})}
\end{aligned}
$$

O precipitado obtido foi mantido em descanso por $24 \mathrm{~h}$. O material decantado foi separado do restante por meio de filtração com bomba de vácuo. $\mathrm{O}$ material foi lavado até a total retirada do subproduto formado $\left(\mathrm{NH}_{4} \mathrm{Cl}\right)$, que foi confirmada por meio de teste negativo com solução de nitrato de prata $\left(\mathrm{AgNO}_{3}\right)$. O precipitado livre de íons cloreto foi seco em estufa por $\sim 24 \mathrm{~h}$ a $105^{\circ} \mathrm{C}$. O material obtido foi então desaglomerado em almofariz de ágata e pesado em balança analítica para se obter o rendimento da reação de síntese. Os reagentes foram pesados e as soluções foram preparadas para a obtenção de $30 \mathrm{~g}$ de hidroxiapatita (HAp) com uma razão final $\mathrm{Ca} / \mathrm{P}=1,67$ o que corresponde a uma hidroxiapatita estequiométrica $\mathrm{Ca}_{10}\left(\mathrm{PO}_{4}\right)_{6}(\mathrm{OH})_{2}$. A pesagem mostrou que o rendimento da reação de síntese foi $89,83 \%$, com a obtenção de $26,95 \mathrm{~g}$ de HAp seca. Após a pesagem o material foi passado em peneira de 200 mesh para garantir a homogeneidade das partículas.

Os processos de sinterização foram feitos em forno EDG F-3000. A sinterização foi feita em cadinhos de alumina em diferentes temperaturas $\left(900,1000,1200\right.$ e $\left.1300^{\circ} \mathrm{C}\right)$ por $2 \mathrm{~h}$. $\mathrm{O}$ tratamento térmico foi conduzido sob atmosfera oxidante a uma taxa de aquecimento e resfriamento de $10{ }^{\circ} \mathrm{C} / \mathrm{min}$. O material sinterizado foi caracterizado por difração de raios $\mathrm{X}$ (DRX), espectroscopia no infravermelho com transformada de Fourier (IVTF), microscopia eletrônica de varredura (MEV), análise termogravimétrica (ATG), análise térmica diferencial (ATD), área de superfície específica (ASE), e o diâmetro médio dos poros foram analisados por meio dos métodos Brunauer-Emmett-Teller (BET) e Barret-JoynerHalenda (BJH), respectivamente.

Os difratogramas de raios $\mathrm{X}$ foram obtidos no difratômetro Shimadzu XRD 6000, utilizando radiação Cuk $\alpha, \lambda=1,542 \mathrm{~nm}$, com varredura de $10-802 \theta$ e operando a $30 \mathrm{kV}$ e $30 \mathrm{~mA}$, com passo de $2^{\circ} \cdot \mathrm{min}^{-1}$.

Os grupos químicos foram identificados por meio de espectroscopia no infravermelho com transformada de Fourier (IVTF) com um espectrômetro Perkin Elmer Spectrum Gx2000. As amostras foram analisadas na forma de pastilhas com $\sim$ 1:300 de hidroxiapatita em KBr. Os espectros foram obtidos pela média de 16 ciclos de varredura e resolução de $4 \mathrm{~cm}^{-1}$.
O estudo da morfologia dos pós se deu a partir de micrografias obtidas pela microscopia eletrônica de varredura em microscópio Zeiss Evo Ma15.

Análises termogravimétrica e térmica diferencial foram feitas nas amostras de hidroxiapatita para se avaliar as reações que ocorrem durante o tratamento térmico em aparelho TA Instr. SDT-2690. O material foi aquecido a uma taxa de $10{ }^{\circ} \mathrm{C} \cdot \mathrm{min}^{-1}$ em um fluxo de ar de $100 \mathrm{~mL} \cdot \mathrm{min}^{-1}$, da temperatura ambiente $\left(\sim 25^{\circ} \mathrm{C}\right)$ até $1300^{\circ} \mathrm{C}$. Como material de referência utilizou-se alumina $\left(\alpha-\mathrm{Al}_{2} \mathrm{O}_{3}\right)$.

A avaliação da área de superfície específica foi feita em um aparelho Quantachrome Nova-2200, por meio de adsorção de nitrogênio pelo método BET e calculada posteriormente por meio do programa Nova Enhanced Data Reduction Software Ver. 2.13@). Os diâmetros dos poros e os volumes médios foram calculados empregando-se o método $\mathrm{BJH}$. Todas as amostras passaram por um pré-tratamento térmico para a remoção de água e produtos voláteis que pudessem estar adsorvidos na superfície da HAp impedindo a posterior adsorção de moléculas de nitrogênio.

\section{RESULTADOS E DISCUSSÃO}

\section{Difração de raios $X$}

Os difratogramas obtidos mostram que a fase predominante é da hidroxiapatita. A indexação dos picos de difração foi realizada com o banco de dados do International Centre for Diffraction Data 00-055-0592. A comparação dos picos das amostras sintetizadas com os padrões presentes na literatura mostra com clareza a efetividade da síntese e a cristalinidade do material obtido [17, 24, 33, 34]. Picos característicos da HAp hexagonal foram observados em $25,8.31,8,32,2,32,9,39,9,46,8,48,2,49,6,50,5,51,3,52,1$ e 53,2 20. Na Fig. 1 é possível observar os difratogramas das amostras sinterizadas e com os planos de difração indexados.

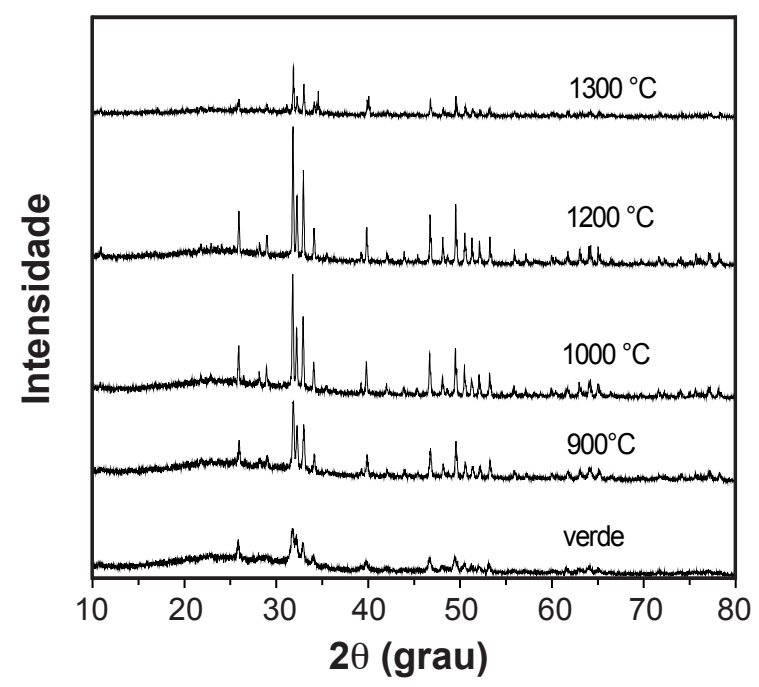

Figura 1: Difratogramas de raios $X$ das amostras de HAp Sin sinterizadas em diferentes temperaturas.

[Figure 1: XRD patterns of samples Sin Hap sintered at different temperature.] 
Estes e outros picos de menor intensidade comprovam que a hidroxiapatita se apresenta como fase majoritária e concordam com os dados encontrados em outros trabalhos $[24,26]$.

Na Fig. 2 é possível observar que com o aumento da temperatura de sinterização a hidroxiapatita se decompõe em fosfato tetracálcico TTCP $\left(\mathrm{Ca}_{4}\left(\mathrm{PO}_{4}\right)_{2} \mathrm{O}\right)$, fosfato tricálcico TCP $\left(\mathrm{Ca}_{3}\left(\mathrm{PO}_{4}\right)_{2}\right)$, pentóxido de fósforo $\left(\mathrm{P}_{2} \mathrm{O}_{5}\right)$ e óxido de cálcio $(\mathrm{CaO})$, como mostrado nas equações $\mathrm{B}$ e $\mathrm{C}$ e pelas novas fases identificadas e indexadas no difratogramas da amostra sinterizada a $1300^{\circ} \mathrm{C}[13,28]$.

$$
\begin{aligned}
& \mathrm{Ca}_{10}\left(\mathrm{PO}_{4}\right)_{6}(\mathrm{OH})_{2} \rightarrow 3 \beta-\mathrm{TCP}+\mathrm{Ca}_{4}\left(\mathrm{PO}_{4}\right)_{2} \mathrm{O}+\mathrm{H}_{2} \mathrm{O} \\
& \mathrm{Ca}_{4}\left(\mathrm{PO}_{4}\right)_{2} \mathrm{O} \rightarrow 4 \mathrm{CaO}+\mathrm{P}_{2} \mathrm{O}_{5} \uparrow
\end{aligned}
$$

As intensidades dos picos mostram que a cristalinidade do mineral aumenta com o aumento da temperatura de sinterização. Esses resultados foram também obtidos por outros autores, em que as intensidades dos picos aumentam com o acréscimo da temperatura do tratamento térmico $[35,36]$. As amostras de hidroxiapatita aumentaram a cristalinidade de $22 \%$ para as amostras verdes para $95 \%$ quando sinterizadas a $1000{ }^{\circ} \mathrm{C}$ por $1 \mathrm{~h}$ [36]. O aumento da cristalinidade veio acompanhado da diminuição da área superficial específica de $170 \mathrm{~m}^{2} / \mathrm{g}$ para $5 \mathrm{~m}^{2} / \mathrm{g}$. As alterações da área de superfície específica com o aumento da temperatura de sinterização também foram estudadas nesse trabalho e os resultados são mostrados mais adiante. A partir dos difratogramas obtidos foi possível se estimar a porcentagem de cristalinidade e o tamanho médio dos cristalitos [12].

$$
\mathrm{Xc} \approx 1-\left(\left(\mathrm{V}_{\frac{112}{300}} / \mathrm{I}_{300}\right) \times 100\right.
$$

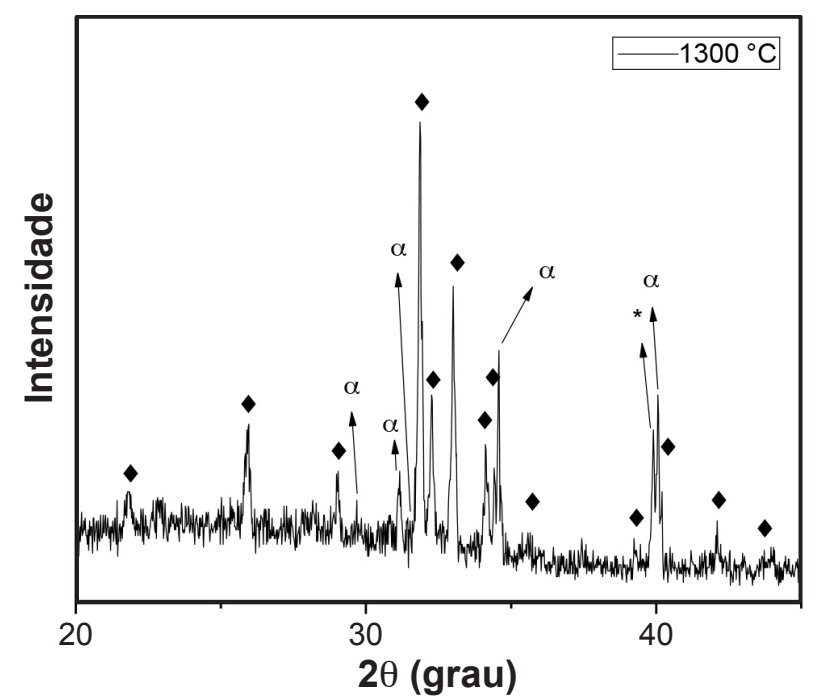

Figura 2: Difratograma de raios X dos produtos da decomposição da HAp Sin sinterizada a $1300{ }^{\circ} \mathrm{C}$ por 2 h. HAp $(\star) \alpha-\mathrm{TCP}(\alpha) \mathrm{CaO}(*)$ [Figure 2: XRD pattern of the product of decomposition Sin HAp sintered at $1300^{\circ} \mathrm{C}$ by 2 h. HAp ( $) \alpha-T C P(\alpha) C a O(*)$.]
Xc é a aporcentagem de cristalinidade da amostra, $\mathrm{V}_{112 / 300}$ é o vale existente entre os picos do difratograma referentes aos planos (112) e (300) e $\mathrm{I}_{300}$ é a intensidade do pico referente ao plano (300).

$$
\mathrm{L}=\frac{\mathrm{k} \lambda}{\beta \cos \theta}
$$

em que L é o tamanho médio dos cristalitos, $\mathrm{K}$ é uma constante que depende da forma dos cristais, que, nesse caso, corresponde a 0,89 . $\beta$ é o valor da largura a meia altura do pico de difração e $\theta$ é o ângulo de Bragg do pico difratado. Para a obtenção do tamanho médio foi utilizado o pico referente ao plano (002) em $\sim 25,82 \theta$. Essa escolha é comumente feita por vários autores com o intuito de evitar a sobreposição de picos próximos, evitando assim a obtenção errônea do tamanho médio dos cristalitos [37].

$\mathrm{O}$ processo de sinterização promoveu o crescimento dos cristalitos e aumentou a cristalinidade dos pós de HAp como observado na Tabela I. O deslocamento dos átomos promoveu o crescimento dos cristais e também favoreceu o reordenamento das células unitárias, aumentando a cristalinidade da matriz cerâmica. Os resultados da Tabela I mostram que um aumento radical do tamanho de cristalitos ocorreu entre 1200 e $1300{ }^{\circ} \mathrm{C}$ o que pode ser relacionado ao início da desidroxilação, o que ocasionou posteriormente a decomposição da hidroxiapatita como mostrado nas equações B e C $[12,38]$. Esses resultados estão de acordo com outros encontrados em trabalhos sobre comportamento térmico da HAp [12, 28, 39].

Tabela I - Valores de tamanho de cristalito e porcentagem de cristalinidade.

[Table I - Values of the crystallite size and percentage of crystallinity.]

\begin{tabular}{ccc}
\hline Amostra & $\begin{array}{c}\text { Cristalinidade } \\
(\%)\end{array}$ & $\begin{array}{c}\text { Tamanho } \\
(\mathrm{nm})\end{array}$ \\
\hline HAp Sin verde & 32,7 & 33,83 \\
HAp Sin $900^{\circ} \mathrm{C}$ & 53,45 & 55,19 \\
HAp Sin $1000^{\circ} \mathrm{C}$ & 73,01 & 67,15 \\
HAp Sin $1200^{\circ} \mathrm{C}$ & 78,95 & 71,95 \\
HAp Sin $1300^{\circ} \mathrm{C}$ & 75,89 & 140,01 \\
\hline
\end{tabular}

\section{Espectroscopia no infravermelho (IVTF)}

A Fig. 3 mostra que os espectros no infravermelho apresentam bandas correspondentes aos grupos fosfato $\left(\mathrm{PO}_{4}{ }^{3-}\right)$, hidroxila $\left(\mathrm{OH}^{-}\right)$, carbonato $\left(\mathrm{CO}_{3}{ }^{2-}\right)$ e também bandas referentes a moléculas de $\mathrm{H}_{2} \mathrm{O}$ fisicamente adsorvidas na superfície da hidroxiapatita. Bandas em $3467 \mathrm{~cm}^{-1}$ e 634 $\mathrm{cm}^{-1}$ são correspondentes ao grupo $\mathrm{OH}^{-}$que caracterizam o composto hidroxiapatita. Bandas correspondentes ao grupo $\mathrm{PO}_{4}^{3-}$ em 1087 e $1030 \mathrm{~cm}^{-1}$ são atribuídas ao estiramento assimétrico $\left(v_{3}\right), 962 \mathrm{~cm}^{-1}$ estiramento simétrico $\left(v_{1}\right)$, deformação angular assimétrica $\left(v_{4}\right)$ em 603 e $564 \mathrm{~cm}^{-1} \mathrm{e}$, em $475 \mathrm{~cm}^{-1}$ angular simétrica $\left(\mathrm{v}_{2}\right)$. Essas bandas relacionadas 
ao grupamento fosfato reforçam a obtenção do composto hidroxiapatita. $\mathrm{O}$ aumento da intensidade dessas bandas está relacionado à maior cristalinidade do material, como foi observado com o aumento da temperatura de sinterização e apresentado na Tabela I [23]. Bandas com comprimentos de onda próximos de 3449 e $1640 \mathrm{~cm}^{-}$ ${ }^{1}$ correspondem a moléculas de água fisicamente ligadas à superfície da HAp [26, 33, 37].

A presença dos íons carbonato nas amostras de hidroxiapatita foi confirmada pelas bandas em 1450 e $1410 \mathrm{~cm}^{-1}$ que foram relacionadas a formação de hidroxiapatita do tipo $\mathrm{B}\left(\mathrm{CO}_{3}{ }^{2-} \rightarrow \mathrm{PO}_{4}{ }^{3-}\right)$. A banda observada em $1540 \mathrm{~cm}^{-1}$ é relacionada à presença de hidroxiapatita do tipo $\mathrm{AB}$, em que os íons $\mathrm{CO}_{3}{ }^{2-}$ ocupam as posições dos íons fosfato e dos íons hidróxido [40]. As bandas dessas espécies têm suas intensidades diminuídas com o aumento da temperatura, atingindo a total descarbonização da HAp em $1100{ }^{\circ} \mathrm{C}[40,41]$. Bandas referentes à espécie cloreto $\left(\mathrm{Cl}^{-}\right)$não foram observadas nos espectros, confirmando a efetividade do processo de lavagem do precipitado.

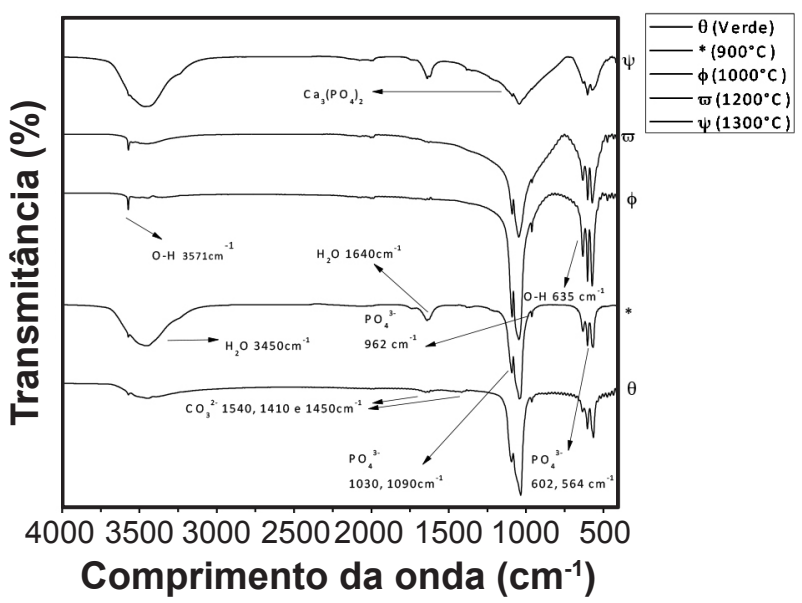

Figura 3: Espectros no infravermelho de amostras de HAp Sin sinterizados em diferentes temperaturas.

[Figure 3: Infrared spectra of samples Sin Hap sintered at different temperatures.]

A HAp tem bandas de $\left(\mathrm{OH}^{-}\right)$diminuídas com o aumento da temperatura de sinterização. Em $1300{ }^{\circ} \mathrm{C}$ a banda em $634 \mathrm{~cm}^{-1}$ é inexistente e a banda em $3467 \mathrm{~cm}^{-1}$ possui a intensidade diminuída. Essa diminuição da intensidade está associada ao início da decomposição do material, que, de acordo com a equação $\mathrm{B}$, promove a desidroxilação da hidroxiapatita e a formação de fosfatos de cálcio mais simples [30]. A decomposição da HAp em outros fosfatos de cálcio pode ser observada pelo alargamento das bandas referentes ao grupamento $\mathrm{PO}_{4}^{3-}$ entre 950 e $1200 \mathrm{~cm}^{-1}$ [30]. Esse alargamento é devido à formação de outros fosfatos que absorvem energia em regiões próximas a do fosfato apatítico e consequentemente promovem a sobreposição de bandas com o posterior alargamento das mesmas, como observado nos espectros das amostras sinterizadas a $1300{ }^{\circ} \mathrm{C}[30]$. ATD

Análise termogravimétrica e térmica diferencial (ATG/

Os resultados das análises de ATG/ATD são apresentados na Fig. 4. A amostra de hidroxiapatita apresenta uma diminuição da massa inicial próxima de $100{ }^{\circ} \mathrm{C}$, que está relacionada à perda da água fisicamente adsorvida na superfície da matriz cerâmica com a consequente absorção de energia (reação endotérmica) [33]. O contínuo aumento da temperatura promove uma acentuada perda de massa próxima entre 150 e $1000{ }^{\circ} \mathrm{C}$, associada ao início da descarbonização do material. Em $\sim 1100{ }^{\circ} \mathrm{C}$ tem o início da decomposição do material e em $1250{ }^{\circ} \mathrm{C}$ a curva ATD exotérmica corresponde à transição de fases do produto da decomposição da hidroxiapatita. Como apresentado na equação $\mathrm{B}$, o $\beta$-TCP proveniente da decomposição da HAp altera sua fase trigonal para a fase monoclínica do $\alpha$-TCP, sendo apresentado por uma curva acima do eixo y do gráfico ATD [13, 26, 28]. A perda de massa observada em temperaturas superiores a $1150{ }^{\circ} \mathrm{C}$ está associada com a perda de água estrutural da hidroxiapatita. Essa perda se dá pelo processo de desidroxilação responsável pela decomposição da HAp [28]. Como observado nos espectros de infravermelho, a diminuição da intensidade das bandas correspondentes ao grupo $\mathrm{OH}^{-}$ocorre concomitantemente ao alargamento das bandas do grupo $\mathrm{PO}_{4}^{3-}$. Tal resultado reforça a ideia de que o início da decomposição da hidroxiapatita em temperaturas próximas de $1200{ }^{\circ} \mathrm{C}$ está relacionado com a perda de água estrutural da matriz (desidroxilação).

\section{Microscopia eletrônica de varredura (MEV)}

As Figs. 5, 6, 7, 8 e 9 apresentam as imagens obtidas por MEV das amostras de HAp. É possível observar na Fig. 5 que os pós não calcinados (verde) apresentam-se como grandes aglomerados, porém, na Fig. 6 observa-

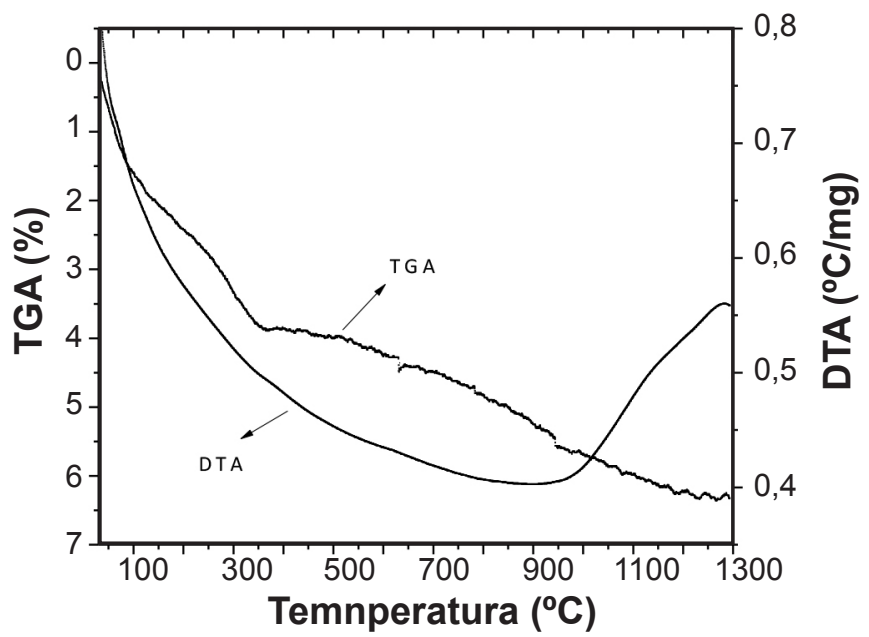

Figura 4: Curvas termogravimétrica e térmica diferencial da amostra de HAp Sin.

[Figure 4: Curve of thermogravimetric and differential thermal analysis of Sin Hap.] 
se que a morfologia da hidroxiapatita se modifica após o tratamento térmico a $900{ }^{\circ} \mathrm{C}$. Nota-se a formação de partículas esféricas, as quais foram obtidas também por outros autores $[12,28]$. As imagens mostram que a sinterização da hidroxiapatita ocorre dentro da faixa de temperatura escolhida. A confirmação da sinterização pode ser observada pela formação de pescoços entre as partículas com posterior crescimento. Nas imagens 7 e 8 é possível observar partículas unidas e maiores do que as visualizadas nas amostras verde e HAp Sin 900. O processo de sinterização dos pós de HAp pode ser acelerado devido a grande área superficial disponível nas amostras verdes e também devido a presença de íons como $\mathrm{CO}_{3}{ }^{2-}[12,38$, 42]. Em temperaturas próximas de $900{ }^{\circ} \mathrm{C}$ os íons $\mathrm{CO}_{3}{ }^{2-}$ são eliminados na forma de $\mathrm{CO}_{2}$ e consequentemente deixam lacunas na estrutura cristalina, facilitando a difusão atômica durante o processo de sinterização e promovendo o crescimento das partículas, diminuição da área de superfície específica e uma consequente densificação aparente da hidroxiapatita [12, 42]. Os íons carbonato somente influenciam no processo de sinterização quando estes ocupam posições dos íons fosfato, formando consequentemente HAp do tipo B. Bandas de vibrações correspondentes a esta substituição foram observadas nos resultados de espectroscopia no infravermelho correlacionando-se dessa maneira com a acelerada modificação da morfologia da hidroxiapatita [12]. Na Fig. 9 é apresentada a imagem do material sinterizado a $1300{ }^{\circ} \mathrm{C}$. A modificação radical da morfologia é observada após esse tratamento térmico. Os resultados estão de acordo com os observados [12, 38], em que as amostras verdes com baixa cristalinidade, grande área de superfície específica e menores tamanhos de cristalitos apresentaram uma densidade próxima de $93 \%$ após a sinterização em $1250{ }^{\circ} \mathrm{C}$. Além disso, a presença de íons carbonato pode ter contribuído no processo de sinterização dos pós aumentando sua eficiência e promovendo a consolidação da hidroxiapatita em menores temperaturas [38].

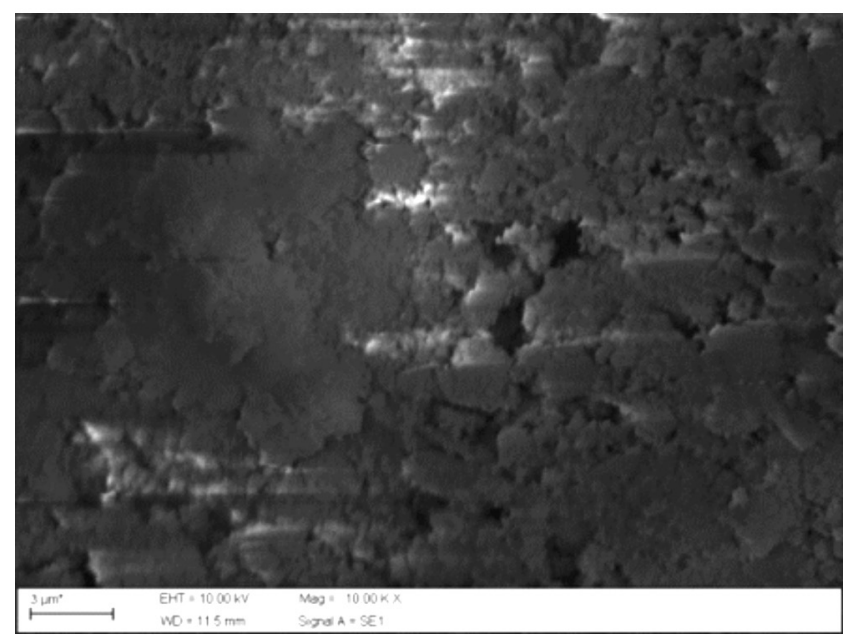

Figura 5: Micrografia obtida por microscopia eletrônica de varredura de HAp Sin verde.

[Figure 5: SEM micrograph of HAp green sample.]

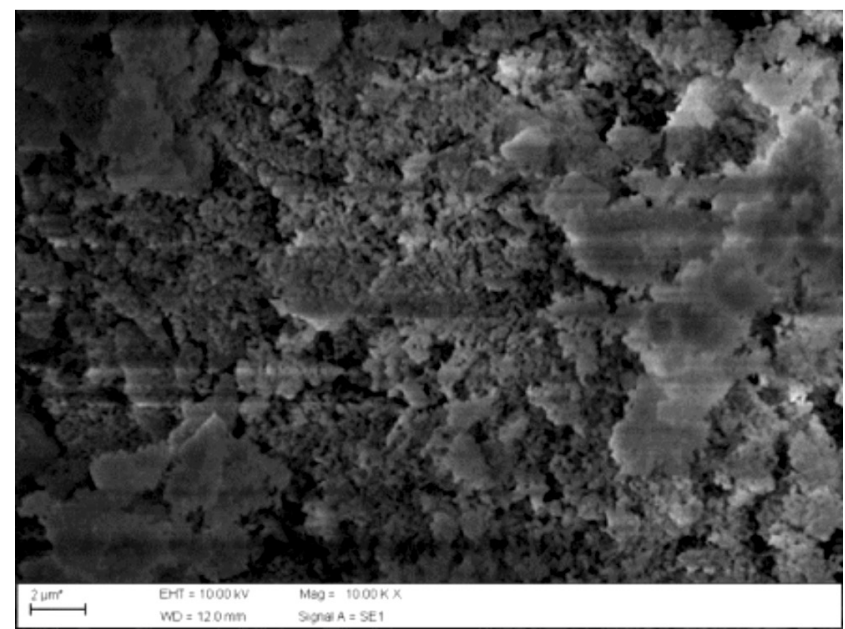

Figura 6: Micrografia obtida por microscopia eletrônica de varredura da amostra HAp $\operatorname{Sin} 900^{\circ} \mathrm{C}$.

[Figure 6: SEM micrograph of HAp sample Sin $900^{\circ} \mathrm{C}$.]

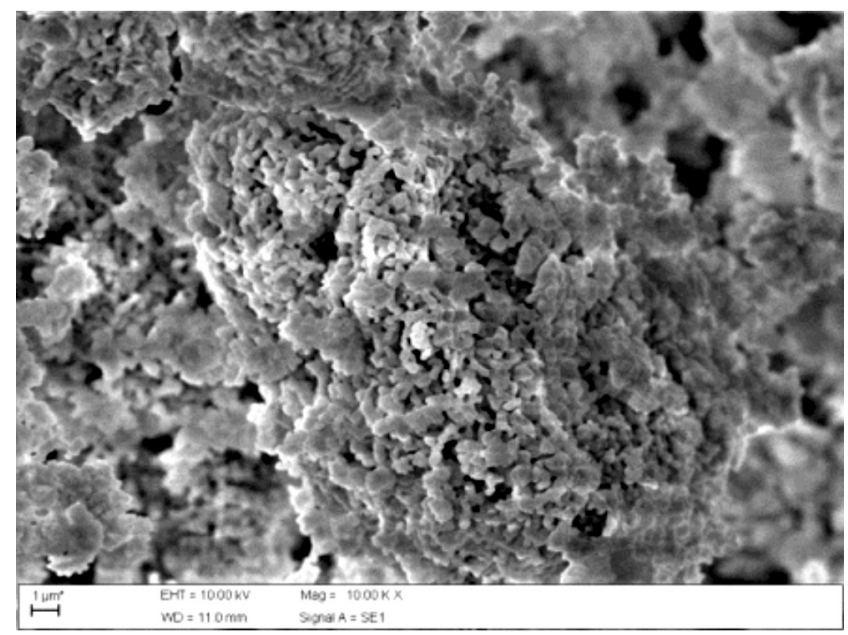

Figura 7: Micrografia obtida por microscopia eletrônica de varredura da amostra HAp Sin $1000^{\circ} \mathrm{C}$.

[Figure 7: SEM micrograph of HAp sample Sin $1000^{\circ} \mathrm{C}$.]

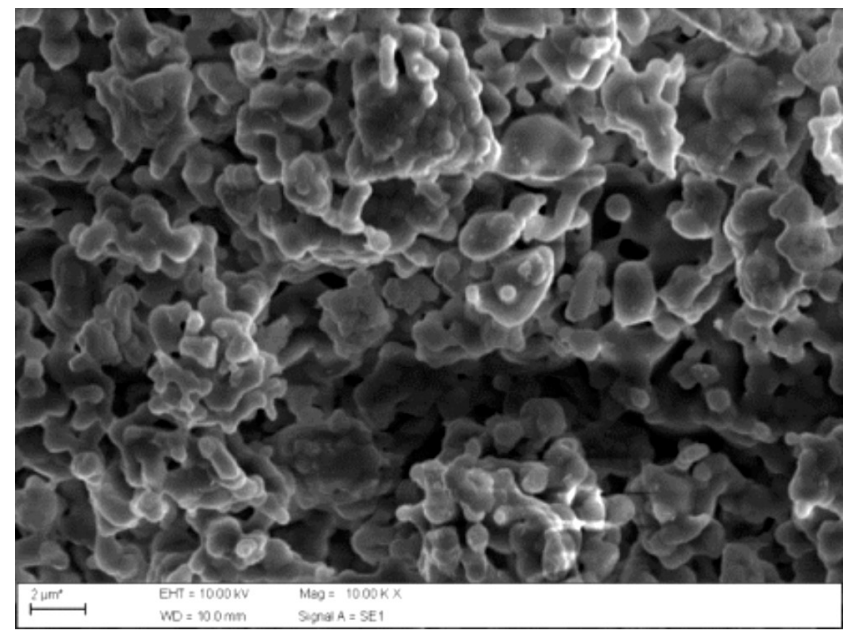

Figura 8: Micrografia obtida por microscopia eletrônica de varredura da amostra HAp $\operatorname{Sin} 1100{ }^{\circ} \mathrm{C}$.

[Figure 8: SEM micrograph of HAp sample Sin $1100^{\circ} \mathrm{C}$.] 


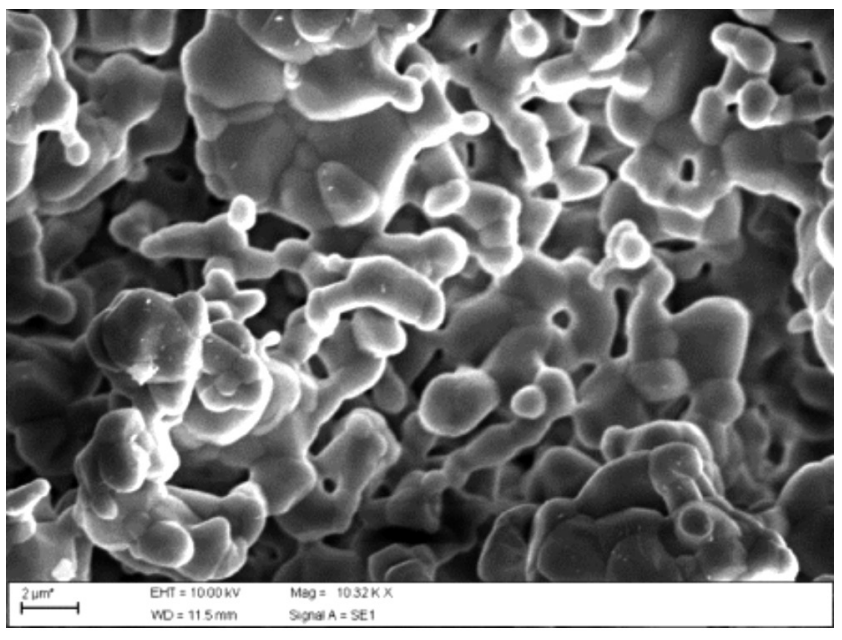

Figura 9: Micrografia obtida por microscopia eletrônica de varredura da amostra HAp Sin $1200{ }^{\circ} \mathrm{C}$.

[Figure 9: SEM micrograph of HAp sample Sin $1200^{\circ} \mathrm{C}$.]

Área de superficie especifica e porosidade (BET e BJH)

O crescimento dos grãos, como observado nas imagens de MEV e pelos cálculos utilizando a equação de DebyeScherrer, foi consequência dos processos de sinterização que iniciaram em temperaturas próximas de $900{ }^{\circ} \mathrm{C}$. Essa afirmação pode ser confirmada pelo resultado da (ASE) que teve uma diminuição dos valores com o aumento da temperatura do tratamento térmico. Os resultados dessa análise são apresentados na Tabela II. Com o aumento da temperatura do tratamento térmico, a área de superfície específica das amostras experimentou uma diminuição de $59,9 \mathrm{~m}^{2} / \mathrm{g}$ para a amostra não calcinada (verde) atingindo valores próximos de $0,0048 \mathrm{~m}^{2} / \mathrm{g}$ após o tratamento a $1300{ }^{\circ} \mathrm{C}$ por $2 \mathrm{~h}$.

A força máxima do processo de sinterização dos pós de HAp tem início em temperaturas próximas de $1100{ }^{\circ} \mathrm{C}$ [12], o que está de acordo com o os resultados encontrados nesse trabalho. Em temperaturas próximas de $1100{ }^{\circ} \mathrm{C}$ ocorreu a diminuição da ASE e concomitantemente o aumento da cristalinidade e tamanho dos cristalitos, aumento da intensidade das vibrações relacionadas ao grupamento fosfato (relacionado ao aumento da cristalinidade da hidroxiapatita) e modificação da morfologia dos pós tendendo a uma densificação, demonstrando que o processo de sinterização foi o responsável pelas mudanças da hidroxiapatita. $\mathrm{O}$ diâmetro dos poros aumenta, pois os poros presentes nos contornos de grão se fundem e são deslocados para outras regiões do corpo, permitindo dessa maneira, o crescimento dos cristalitos [28] (Tabela II). Embora o diâmetro dos poros aumente, o volume médio destes se apresenta com valores muito pequenos. Essa diminuição pode estar associada à diminuição da área de superfície específica das amostras tratadas a $1300^{\circ} \mathrm{C}$.

\section{CONCLUSÕES}

Os pós de hidroxiapatita foram obtidos pela precipitação das soluções aquosas, sendo as fases e bandas referentes ao material confirmados por análises de difração de raios $\mathrm{X}$ espectroscopia no infravermelho, respectivamente. $\mathrm{O}$ material apresentou modificações cristalinas, morfológicas e texturais após o processo de sinterização. Em temperaturas elevadas, novas fases correspondentes à decomposição da hidroxiapatita foram identificadas. Modificações nas bandas dos espectros corroboraram com os resultados obtidos pela difração de raios $\mathrm{X}$, confirmando a decomposição dos pós em temperaturas de sinterização próximas de 1300 ${ }^{\circ} \mathrm{C}$. Crescimento dos grãos, aumento da cristalinidade e diminuição da área superficial específica também ocorreram quando as amostras foram sinterizadas, mostrando a importância do controle desse processo durante a produção de pós de hidroxiapatita. O entendimento de todos os processos envolvidos durante a sinterização dos pós de hidroxiapatita é importante, pois o processo se faz necessário para consolidar a obtenção do material, trazendo modificações na matriz cerâmica e promovendo alterações nas propriedades físicas e químicas dos pós.

\section{AGRADECIMENTOS}

Programa de Pós-Graduação em Física e Química de Materiais - FQMat, Universidade Federal de S. J. del-Rei - UFSJ, Fundação de Amparo à Pesquisa de Minas Gerais - FAPEMIG e Universidade Federal de Itajubá - UNIFEI.

\section{REFERÊNCIAS}

[1] D. Gouveia, A. Bressiani, J. Bressiani, XVII CBECImat, Foz Do Iguaçu, PR (2006) 133.

Tabela II - Resultados de área superficial específica e diâmetro médio dos poros de amostras de HAp Sin quantificadas pelo método BET e BJH.

[Table II - Results of specific surface area and average pore diameter of samples of HAp Sin quantified for BET and BJH method.]

\begin{tabular}{cccc}
\hline Amostra & $\begin{array}{c}\text { Área superficial específica } \\
\left(\mathrm{m}^{2} / \mathrm{g}\right)\end{array}$ & $\begin{array}{c}\text { Diâmetro médio dos poros } \\
(\AA)\end{array}$ & $\begin{array}{c}\text { Volume total dos poros } \\
\left(\mathrm{cm}^{3} / \mathrm{g}\right)\end{array}$ \\
\hline HAp Sin verde & 59,9 & 182,1 & 0.273 \\
$\mathrm{HAp} \mathrm{Sin} 900^{\circ} \mathrm{C}$ & 19,4 & 110,0 & 0.048 \\
$\mathrm{HAp} \mathrm{Sin} 1300{ }^{\circ} \mathrm{C}$ & 0,0048 & 333,9 & 0.00004 \\
\hline
\end{tabular}


[2] M. C. Valente, "Síntese de hidroxiapatita e sua aplicação como biomaterial", Diss. Mestrado, Univ. de S. Paulo, Brasil (1999).

[3] A. H. dos S. Rebelo, "Preparação de hidroxiapatita nanométrica com características adequadas ao processamento coloidal para aplicações biomédicas", Diss. Mestrado, Univ. de Aveiro, Portugal (2006).

[4] A. Pereira, W. Vasconcelos, R. Oréfice, Polim.: Ci. Tecnol. (1999) 104.

[5] S. Raynaud, E. Champion, D. Bernache-Assollant, P. Thomas, Biomater. 23, 4 (2002) 1065.

[6] J. Gustavsson, M. P. Ginebra, E. Engel, J. Planell, Acta Biomater. 7 (2011) 4242.

[7] E. Meurice, A. Leriche, J. Hornez, F. Bouchart, R. Emmanuelle, B. Laurent, D. Michel, C. Francis. J. Eur. Ceram. Soc. 32 (2012) 2673.

[8] S. Sugiyama, N. Fukuda, H. Matsumoto, H. Hayashi, N. Shigemoto, Y. Hiraga, J. Moffat, J. Colloid Interface Sci. 220 (1999) 324.

[9] B. Li, X. Chen, B. Guo, X. Wang, H. Fan, X. Zhang, Acta Biomater. 5 (2009) 134.

[10] J. P. Lafon, E. Champion, D. Bernache-Assollant, J. Eur. Ceram. Soc. 28 (2008) 139.

[11] T. I. Ivanova, O. V. Frank-Kamenetskaya, A. B. Kol'tsov, V. L. Ugolkov, J. Solid State Chem. 160 (2001) 340.

[12] E. Landi, A. Tampieri, G. Celotti, S. Sprio, J. Eur. Ceram. Soc. 20 (2000) 2377.

[13] E. Champion, Acta Biomater. 9 (2013) 5855.

[14] M. Jiménez-Reyes, M. Solache-Ríos, J. Hazardous Mater. 180 (2010) 297.

[15] S. Bailliez, A. Nzihou, E. Beche, Process Safety Environ. Protec. 82 (2004) 175.

[16] A. K. Nayak, Int. J. Chem. Tech. Res. 2 (2010) 903.

[17] S. Madhavi, C. Ferraris, T. J. White, J. Solid State Chem. 178 (2005) 2838.

[18] B. Mavis, A. Cu, J. Am. Ceram. Soc. 91 (2000) 989.

[19] C. Garcia, P. Carlos, J. Gaviria, DYNA 73 (2006) 9.

[20] A. C. F. M. Costa, M. G. Lima, L. H. M. A. Lima, V. V. Cordeiro, K. M. S. Viana, Rev. Eletr. Mater. Proc. 3 (2009) 29.

[21] Y. Zhang, Y. Yokogawa, X. Feng, Y. Tao, Y. Li, Ceram. Int. 36 (2010) 107.

[22] S. M. Cunha, D. R. R. Lazar, V. Ussui, N. B. Lima, A. H. A. Bressiani, XVI CBECImat, Porto Alegre, RS (2004).
[23] G. E. J. Poinern, M. K. Ghosh, Y. -J. Ng, T. B. Issa, S. Anand, P. Singh, J. Hazardous Mater. 185 (2011) 29.

[24] M. Aminzare, A. Eskandari, M. H. Baroonian, A. Berenov, Z. Razavi Hesabi, M. Taheri, S. K. Sadrnezhaad, Ceram. Int. 39 (2013) 2197.

[25] B. Henrique, G. Lourenço, E. De Sousa, G. Silva, IX Enc. Lat. Am. Pós Graduação (2011) 1.

[26] S. Ramesh, K. L. Aw, R. Tolouei, M. Amiriyan, C. Y. Tan, M. Hamdi, J. Purbolaksono, M. A. Hassan. W.D. Teng, Ceram. Int. 39, 1 (2013) 111.

[27] A. G. S. Azevedo, Rev. Univ. Vale do Rio Verde 10 (2012) 297.

[28] S. F. Ou, S.Y. Chiou, K. L. Ou, Ceram. Int. 39 (2013) 3809.

[29] Y. Sung, J. Lee, J. Yang, J. Crystal Growth 262 (2004) 467.

[30] C. J. Liao, F. H. Lin, K. S. Chen, J. S. Sun, Biomater. 20 (1999) 1807.

[31] M. H. Santos, M. de Oliveira, L. Palhares, D. F. Souza, H. S. Mansur, W. L. Vasconcelos, Mater. Res. 7 (2004) 625. [32] B. Li, X. Chen, B. Guo, X. Wang, H. Fan, X. Zhang, Acta Biomater. 5 (2009) 134.

[33] M. Markovic, B. O. Fowler, M. S. Tung, J. Res. Nat. Inst. Stand Technol. 109 (2004)553.

[34] M. L. Santos, A. O. Florentino, M. J. Saeki, A. H. Aparecida, M. V. L. Fook, A. C. Guastaldi, Ecl. Quim. 30 (2005) 29.

[35] S. Gao, R. Sun, Z.Wei, H. Zhao, H. Li, F. Hu, J. Fluorine Chem. 130 (2009) 550.

[36] C. Stötzel, F. A. Müller, F. Reinert, F. Niederdraenk, J. E. Barralet, U. Gbureck, Colloids Surfaces B, Biointerfaces 74 (2009) 91.

[37] S. Kongsri, K. Janpradit, K. Buapa, S. Techawongstien, S. Chanthai, Chem. Eng. J. 215 (2013) 522.

[38] A. Tampieri, G. Celotti, S. Sprio, C. Mingazzini, Mater. Chem. Phys. 34 (2000) 54.

[39] J. Song, Y. Liu, Y. Zhang, L. Jiao, Mater. Sci. Eng. 528 (2011) 5421.

[40] A. Ślósarczyk, Z. Paszkiewicz, C. Paluszkiewicz, J. Molecular Structure 744 (2004) 657.

[41] A. Shaltout, M. Allam, M. Moharram, Spectrochim. Acta, Part A: Molecular and Biomolecular Spectroscopy 83 (2011) 56.

[42] Y. Boiko, H. Worch, H. Science of Sintering 31, (1999) 151.

(Rec. 29/08/2014, Ac. 02/11/2014) 